\section{Submitted: 09.03.2020 Accepted: \\ Shear wave elastography reveals a high prevalence of liver fibrosis in overweight or obese Hispanic youth} 07.07.2020

Published: 28.09.2020

Keywords prediabetes, fibrosis, diabetes, elastography, Hispanic

\author{
Sanjeet Sandhu' ${ }^{1}$, Carisse Orsi ${ }^{1}$, Gary L. Francis ${ }^{1}$, Zhu Wang ${ }^{2}$, \\ Roman Fernandez ${ }^{2}$, Naim Alkhouri ${ }^{3}$ \\ ${ }^{1}$ University of Texas Health and Science Center, Department of Pediatric Endocrinology, \\ San Antonio, TX, United States \\ ${ }^{2}$ University of Texas Health and Science Center Department of Population Health Sciences, \\ San San Antonio TX, United States \\ ${ }^{3}$ Texas Liver Institute and University of Texas Health, Department of Pediatric Gastroenterology, \\ San Antonio, TX, United States \\ Correspondence: Sanjeet Sandhu, 1201 E. Schuster Ave \#4A, El Paso, TX 79902, United States
}

DOI: $10.15557 /$ JoU.2020.0027

\begin{abstract}
Background: Obesity, prediabetes, and type 2 diabetes are risk factors for nonalcoholic fatty liver disease. Inflammation and hepatocellular damage associated with nonalcoholic fatty liver disease lead to progressive non-alcoholic steatohepatitis, fibrosis and cirrhosis. Current tests to identify fibrosis (liver biopsy) are invasive and not conducive to serial examination. For that reason, we used the newer technique of shear wave elastogrophy (SWE) to detect fibrosis in overweight or obese Hispanic youth and sought to determine if carbohydrate tolerance or insulin resistance were associated with fibrosis in this high risk population. Methods: A total of 67 Hispanic youth (8-18 years of age) with overweight or obesity who were referred for multidisciplinary evaluation were included. SWE was used to identify those with suspected fibrosis. Results of SWE were then compared with glycohemoglobin $\left(A_{1 c}\right)$, insulin resistance (homeostatic model of insulin resistance), and biochemical parameters. Results: The prevalence of suspected fibrosis (SWE $>5.10 \mathrm{kPa}$ ) in overweight or obese Hispanic youth was $62.7 \%$ (42/67). Patients with suspected fibrosis ( $\mathrm{SWE} \geq 5.10 \mathrm{kPa}$ ) had significantly higher levels of serum aspartate aminotransferase, alanine aminotransferase and the aminotransferase to platelet ratio index when compared to patients without significant fibrosis $(\mathrm{SWE}<5.01 \mathrm{kPa})$. However, there were no significant differences between the groups in body mass index, $\mathrm{A}_{1 \mathrm{c}}$, or homeostatic model of insulin resistance. Conclusions: SWE detected a high prevalence $(62.7 \%)$ of suspected hepatic fibrosis in a group of high risk, overweight or obese Hispanic youth suggesting that SWE
\end{abstract} is a useful tool for surveillance and longitudinal studies.

\section{Introduction}

Childhood obesity is a worldwide epidemic now affecting more than $20 \%$ of children and adolescents in the United States $^{(1)}$. As the prevalence of obesity has increased, obesity-related nonalcoholic fatty liver disease (NAFLD) has emerged as a common comorbidity in the pediatric population $^{(2,3)}$. Defined as simple steatosis, NAFLD is the leading cause of chronic liver disease in adolescents and young adults. Chronic inflammation and resultant hepatocellular injury then develop as NAFLD progresses into non-alcoholic steatohepatitis (NASH). Up to $40 \%$ of patients (adults and children) with NASH will develop liver fibrosis and eventually cirrhosis ${ }^{(3)}$.

The prevalence of NALFD among obese adolescents is as high as $70-80 \%$ depending on the diagnostic tool used for detection $^{(4)}$. A more granular analysis of these data reveals that the prevalence and course of NAFLD and NASH vary among ethnic and racial groups. Population-based studies 
of adults indicate that males and Hispanics are at highest risk $^{(5)}$. In addition, Hispanics with NAFLD progress into $\mathrm{NASH}$ at a younger age and have a worse prognosis when compared to non-Hispanic whites ${ }^{(6-8)}$.

Type 2 Diabetes (T2DM) is another major risk factor for NAFLD and $\mathrm{NASH}^{(9-11)}$, and the leading cause of liver disease in the United States. Approximately $50 \%$ of adults with T2DM have NAFLD and the prevalence of T2DM among adults with cirrhosis is as high as $57 \%{ }^{(9)}$. Current evidence suggests that insulin resistance may be an important pathogenic factor linking the progression from NAFLD into $\mathrm{NASH}^{(11,12)}$

While the obesity epidemic has prompted increased screening for prediabetes and T2DM in the pediatric population, current screening guidelines fail to screen for NAFLD ${ }^{(13-15)}$. Furthermore, current criteria used to detect NAFLD fail to distinguish patients who are at risk to progress to NASH. Pediatric screening guidelines to evaluate obesity in youth with high BMI include testing for serum alanine aminotransferase (ALT) (a marker for liver inflammation) which is not adequate for assessment of existing NAFLD nor predictive of the risk for NAFLD progression into NASH and cirrhosis $^{(15)}$. Multiple pediatric studies have shown that ALT levels can be normal, even in advanced cirrhosis, leading to missed/delayed opportunities for early intervention ${ }^{(16)}$. Proven adult scoring systems such as aspartate aminotransferase (AST)/ALT ratio, AST/platelet (PLT) ratio index (APRI), NAFLD fibrosis score, and Fibrosis-4 (FIB-4) index also have poor performance in detecting significant fibrosis in children with NAFLD ${ }^{(17)}$. This calls into question the ability of current screening guidelines to identify youth at risk for NAFLD. This is a major public health issue since, left untreated, undiagnosed NAFLD/NASH may eventually progress to cirrhosis with increased morbidity and mortality ${ }^{(2,14)}$.

Liver biopsy is currently the gold standard to identify liver fibrosis, but this procedure is invasive and associated with major complications, which limit the utility of liver biopsy for surveillance and natural history studies. For that reason, many adolescents with liver fibrosis remain undiagnosed and are at increased risk for liver failure ${ }^{(2)}$. The newer and non-invasive imaging techniques of transient elastography (TE) and shear-wave elastography (SWE) provide the opportunity to detect hepatic fibrosis and are a safe and reliable alternative to liver biopsy ${ }^{(18)}$. Furthermore, TE and SWE are reproducible and quantifiable measures of liver stiffness. Using an acoustic pulse, elastography measures liver stiffness (an indirect surrogate measure of fibrosis) in a rapid, reproducible manner that is more reliable than serum biomarkers ${ }^{(19)}$. TE and SWE give an accurate estimation of liver stiffness that correlates with fibrosis staging in pediatric and adolescent patients ${ }^{(13,19,20)}$. Furthermore, the degree of fibrosis directly correlates with long-term prognosis in patients with NAFLD ${ }^{(16)}$.

Although population studies have shown that NASH begins earlier and has a worse prognosis for Hispanics, ${ }^{(6-8)}$ previous studies of adolescent Hispanic cohorts with biopsy proven active fibrosis showed that serum ALT was normal at the time of biopsy, punctuating the need for improved techniques to identify fibrosis in high risk youth ${ }^{(3,7,17)}$. In addition, there are limited data that have examined potential relationships between hepatic fibrosis and glycated hemoglobin $\left(\mathrm{A}_{1 \mathrm{c}}\right)$ in children and adolescents. There is also a paucity of SWE data for high-risk minorities, especially Hispanics, and no consensus as to the optimal SWE score that should be used to distinguish those with suspected fibrosis from those without. A previous study of Italian patients by Nobili et. al. used elastography $\geq 5.10 \mathrm{kPa}$ to suggest the presence of hepatic fibrosis ${ }^{(7)}$. In contrast, a recent study in Korean youth used an SWE cutoff value of $5.50 \mathrm{kPa}$ to identify suspected fibrosis ${ }^{(18)}$.

For these many reasons, we assessed the prevalence of liver fibrosis using SWE in high-risk, overweight and obese Hispanic youth. We then examined potential relationships between SWE, carbohydrate tolerance (glycohemoglobin, $\mathrm{A}_{1 \mathrm{c}}$ ), insulin resistance (homeostatic model of insulin resistance, HOMA-IR) and a variety of biochemical biomarkers, including serum ALT, AST, and APRI.

\section{Methods}

This was a retrospective review of medical records from overweight or obese Hispanic youth referred for multidisciplinary evaluations in the pediatric endocrinology or gastroenterology clinics at the University of Texas Health and Science Center at San Antonio (UTHSCSA) between 2017 and 2019. The study was approved by the Institutional Review Board at UTHSCSA prior to subject enrollment. De-identified patient data were stored securely in a RedCap database.

Patients were eligible for chart review if they were between the ages of $8-18$ years, had a body mass index (BMI) $>85 \%$ for age, and self-identified as being of Hispanic descent.

Patients were excluded from chart review if the serum AST or ALT was $>200 \mathrm{U} / \mathrm{L}$, based on previous studies showing that levels above $200 \mathrm{U} / \mathrm{L}$ and associated inflammation can confound the findings of elastography ${ }^{(21)}$. Patients were also excluded if they were being treated with subcutaneous insulin, glucocorticoids, or angiotensin-converting-enzyme (ACE) inhibitors. Additionally, patients with other causes for chronic liver, renal, or cardiac disease were also excluded.

We reviewed data from 112 potential subjects and identified 67 patients whose records contained both SWE and $A_{1 c}$ levels. These 67 subjects comprise the entire cohort of atrisk subjects. Subjects were first separated into two groups based on SWE score. Group A had no evidence for fibrosis based on SWE $<5.10 \mathrm{kPa}$. Group B had evidence for fibrosis based on $\mathrm{SWE} \geq 5.10 \mathrm{kPa}$. Subjects were then separated into two groups based on $\mathrm{A}_{1 \mathrm{c}}$ using the definition of prediabetes and diabetes from the American Diabetes Association (pre-DM or DM as $\left.\mathrm{A}_{1 \mathrm{c}} \geq 5.7 \%\right)^{(22)}$. Group $\mathrm{C}$ had evidence for pre-DM or DM as shown by $A_{1 c} \geq 5.7 \%$. Group D had normal carbohydrate tolerance as shown by $\mathrm{A}_{1 \mathrm{c}}<5.7 \%$. Additional laboratory and imaging studies were not available for all subjects, but variably included liver ultrasound 
(US), fasting glucose, insulin level, liver enzymes, lipid profile, complete blood count, complete metabolic panel, 25-hydroxy-vitamin D (25OH-D), thyrotropin (TSH), and free-thyroxine (FT4).

Fasting SWE was performed within 6 months of laboratory studies using a Philips brand 2-D shear wave transducer with the C5-1 abdominal convex probe. All measurements were performed in the supine position. Each patient had ten 2D-SWE measurements of the right liver lobe with acceptable interquartile range (IQR) of $30 \%$. The mean of the 10 acceptable measurements and standard deviation within the region of interest were recorded and used to generate the SWE score. The SWE score was expressed in $\mathrm{kPa}$ and a score $\geq 5.10 \mathrm{kPa}$ (based on previous data from Nobili et al. $)^{(7)}$ was used to suggest fibrosis. Suggested fibrosis is the preferred terminology used to identify this group as the definitive procedure of liver biopsy was not performed in all subjects.

Statistical analysis was performed using $\mathrm{R}$ Core Team Statistical Software with a $p$-value $<0.05$ considered statistically significant. The prevalence of suspected fibrosis (SWE $\geq 5.10 \mathrm{kPa}$ ) was then compared between groups using chi-square and non-parametric Wilcoxon rank-sum tests for categorical and numeric variables, respectively. Tests of association were done using Pearson correlations R and linear regression.

\section{Results}

This study cohort of 67 overweight and obese Hispanic youth had a mean age of $13.59 \pm 3.0$ years (range $8-18$ years). Table 1 shows data for the entire cohort, along with separate data for those with and without suspected fibrosis. As shown in Tab. 1, slightly more than half (52.2\%) of the entire cohort were male. Based on SWE scores $\geq 5.10 \mathrm{kPa}$, the prevalence of suspected fibrosis in these obese Hispanic youth was $62.7 \%$ (42/67). There were 25 patients $(37.3 \%)$ with no suspected fibrosis (SWE $<5.10 \mathrm{kPa}$, Group A). Group A had a mean age $(13.12 \pm 2.80$ years, range $8-18$ years $)$, mean BMI-SDS $(2.42 \pm 0.40$, range 1.45-3.37) and mean serum $A_{1 c}$ level (5.71 \pm 074 , range 5.0-7.0) similar to those of the entire cohort. There were 42 patients with suspected fibrosis (SWE $>5.10 \mathrm{kPa}$, Group B). The mean age (13.88 \pm 3.10 years, range $8-18)$, mean BMI-SDS (2.48 \pm 0.38 , range 1.66-3.22), and mean $\mathrm{A}_{1 \mathrm{c}}(5.80 \pm 0.74$, range 4.5-7.9) for group B were also similar to those of the entire group as well as the sub-group without suspected fibrosis (Group A). However, mean ALT in the group without suspected fibrosis $(60.04 \mathrm{U} / \mathrm{L} \pm 41.01$, range 19-181) was significantly lower $(p=0.01)$ than that in the group with suspected fibrosis $(89.27 \mathrm{U} / \mathrm{L} \pm 43.75$, 23-189). Furthermore, there was a tendency for serum ALT to positively associate with SWE score $(\mathrm{R}=0.23)$, but the trend was not significant $(p=0.070)$. Similarly, mean serum AST level in the group without suspected fibrosis

Tab. 1. Demographic and laboratory features of patients with (SWE Score $>5.10 \mathrm{kPa}$ ) and without (SWE Score $<5.1 \mathrm{kPa}$ ) suspected hepatic fibrosis

\begin{tabular}{|c|c|c|c|c|}
\hline & Entire cohort $N=67$ & $\begin{array}{c}\text { Group A } \\
\text { No suspected fibrosis } \\
\text { (SWE score }<5.10 \mathrm{kPa} \text { ) } \\
\begin{array}{c}N=25 \\
\text { Mean } \pm \text { SD (range) }\end{array}\end{array}$ & $\begin{array}{c}\text { Group B } \\
\text { Suspected fibrosis } \\
\text { (SWE score } \geq 5.10 \mathrm{kPa} \text { ) } \\
\begin{array}{c}\mathrm{N}=\mathbf{4 2} \\
\text { Mean } \pm \text { SD (range) }\end{array}\end{array}$ & $\begin{array}{c}P=\text { for Group A } \\
\text { vs. Group B }\end{array}$ \\
\hline Age (yr) & $\begin{array}{l}13.59 \\
(8-18)\end{array}$ & $\begin{array}{c}13.12 \pm 2.80 \\
(8-18)\end{array}$ & $\begin{array}{c}13.88 \pm 3.10 \\
(8-18)\end{array}$ & 0.328 \\
\hline Gender & $\begin{array}{c}\text { Male }=35 \\
\text { Female }=32\end{array}$ & $\begin{array}{c}\text { Male }=15 \\
\text { Female }=10\end{array}$ & $\begin{array}{c}\text { Male }=20 \\
\text { Female }=22\end{array}$ & 0.326 \\
\hline BMI SDS & $\begin{array}{l}2.49 \pm 0.39 \\
(1.45-3.37)\end{array}$ & $\begin{array}{l}2.42 \pm 0.40 \\
(1.45-3.37)\end{array}$ & $\begin{array}{l}2.48 \pm 0.38 \\
(1.66-3.22)\end{array}$ & 0.603 \\
\hline Serum ALT (U/L) & $\begin{array}{c}78.48 \pm 44.75 \\
(19-189)\end{array}$ & $\begin{array}{c}60.04 \mathrm{U} / \mathrm{L} \pm 41.01 \\
(19-181)\end{array}$ & $\begin{array}{c}89.27 \mathrm{U} / \mathrm{L} \pm 43.75 \\
(23-189)\end{array}$ & 0.010 \\
\hline Serum AST (U/L) & $\begin{array}{c}40.1 \pm 22.1 \\
(11-110)\end{array}$ & $\begin{array}{c}31.12 \mathrm{U} / \mathrm{L} \pm 14.92 \\
(14-75)\end{array}$ & $\begin{array}{c}45.37 \mathrm{U} / \mathrm{L} \pm 23.99 \\
(11-110)\end{array}$ & 0.011 \\
\hline$A_{1 c}(\%)$ & $\begin{array}{c}5.77 \pm 0.64 \\
(4.5-7.9)\end{array}$ & $\begin{array}{c}5.71 \pm 074 \\
(5.0-7.0)\end{array}$ & $\begin{array}{c}5.80 \pm 0.74 \\
(4.5-7.9)\end{array}$ & 0.557 \\
\hline SWE Score (kPa) & $\begin{array}{c}5.61 \pm 1.43 \\
(1.47-10.45)\end{array}$ & $\begin{array}{c}4.47 \pm 0.83 \\
(1.47-5.06)\end{array}$ & $\begin{array}{l}6.28 \pm 1.28 \\
(5.2-10.45)\end{array}$ & $<0.001$ \\
\hline HOMA-IR & $\begin{array}{c}10.62 \pm 10.19 \\
(2.84-49.24)\end{array}$ & $\begin{array}{l}14.25 \pm 14.85 \\
(3.38-49.24)\end{array}$ & $\begin{array}{c}8.55 \pm 4.36 \\
(2.84-17.40)\end{array}$ & 0.215 \\
\hline Vitamin D, $250 \mathrm{OH}(\mathrm{ng} / \mathrm{mL})$ & $\begin{array}{c}14.23 \pm 5.44 \\
(6-28)\end{array}$ & $\begin{array}{c}14.60 \pm 5.42 \\
(7-28)\end{array}$ & $\begin{array}{c}14.00 \pm 5.55 \\
(6-25)\end{array}$ & 0.742 \\
\hline Platelets ( $\times 10^{9}$ per liter) & $\begin{array}{c}310 \pm 75.50 \\
(187-490) \\
\end{array}$ & $\begin{array}{c}325.67 \pm 45.40 \\
(276-452)\end{array}$ & $\begin{array}{c}301.70 \pm 81.79 \\
(187-490)\end{array}$ & 0.360 \\
\hline APRI & $\begin{array}{l}0.40 \pm 0.25 \\
(0.12-1.19) \\
\end{array}$ & $\begin{array}{l}0.25 \pm 0.13 \\
(0.12-0.38) \\
\end{array}$ & $\begin{array}{l}0.49 \pm 0.27 \\
(0.16-1.19) \\
\end{array}$ & 0.011 \\
\hline Metformin users & 8 & 2 patients & 6 patients & 0.442 \\
\hline
\end{tabular}


Tab. 2. Demographic and laboratory features of patients who underwent liver biopsy

\begin{tabular}{|c|c|}
\hline & Subjects with liver biopsy $N=5$ \\
\hline Age (yr) & $14.00(8-17)$ \\
\hline Fibrosis stage & $\begin{array}{l}\text { Stage } 1=4 \text { patients } \\
\text { Stage } 2=1 \text { patient }\end{array}$ \\
\hline Gender & $\begin{array}{c}\text { Male }=3 \\
\text { Female }=2\end{array}$ \\
\hline BMI SDS & $2.44 \pm 0.55(1.66-3.11)$ \\
\hline Serum ALT (U/L) & $133.20 \pm 49.70(86-189)$ \\
\hline Serum AST (U/I) & $76.40 \pm 33.20(34-110)$ \\
\hline$A_{1 c}(\%)$ & $6.15 \pm 0.93(5.4-7.4)$ \\
\hline SWE score $(\mathrm{kPa})$ & $7.45 \pm 1.43(6.05-10.45)$ \\
\hline Vitamin D 25OH (ng/mL) & $9.75 \pm 2.22(9-13)$ \\
\hline Platelets $\left(\times 10^{9}\right.$ per liter $)$ & $277.10 \pm 76.07(187-431)$ \\
\hline AST/PLT ratio & $0.64 \pm 0.41(0.33-1.52)$ \\
\hline Metformin users & $N=2$ \\
\hline \multicolumn{2}{|c|}{$\begin{array}{l}\text { BMI SDS - body mass index standard deviation score for age; ALT } \\
\text { - alanine aminotransferase; AST - aspartate aminotransferase; } \text { A }_{1 c}- \\
\text { glycohemoglobin; SWE score - liver stiffness measured in kilopascals } \\
\text { (kPa); HOMA-IR - homeostatic model of insulin resistance; APRI - AST/ } \\
\text { platelet ratio index. NOTE: None of the } 5 \text { patients had a HOMA-IR }\end{array}$} \\
\hline
\end{tabular}

(Group A, 31.12 U/L \pm 14.92 , range 14-75) was significantly lower than that of the group with suspected fibrosis (Group B, $45.37 \mathrm{U} / \mathrm{L} \pm 23.99$, range $11-110, p=0.011$ ). There was also a tendency for serum AST to positively associate with SWE score $(\mathrm{R}=0.22)$, but the trend was not significant ( $p=0.078)$. Finally, the mean APRI was significantly ( $p=0.011)$ lower in the group without suspected fibrosis (Group A, $0.25 \pm 0.13$, range 0.12-0.38) compared to the group with suspected fibrosis (Group B, $0.49 \pm 0.27$, range $0.16-1.19$ ), but there was no significant association between SWE score and APRI $(\mathrm{R}=0.25, p=0.16)$.

As shown in Tab. 2, five patients (all from Group B with suspected fibrosis) underwent liver biopsy for definitive histopathological diagnosis. Fibrosis was staged as follows: Stage 0 = none; Stage 1 = peri-sinusoidal or peri-portal fibrosis; Stage 2 = peri-sinusoidal and portal/peri-portal fibrosis; Stage 3 = bridging fibrosis; and Stage $4=$ cirrhosis. All five were proven to have hepatic fibrosis ranging from stage 1 stage 2 and their SWE scores included some of the highest in the study (mean $=7.45 \pm 1.43$, range 6.05-10.45).

A recent study in Korean youth used an SWE cutoff value of $5.50 \mathrm{kPa}$ to identify suspected fibrosis and found a $50.1 \%$ prevalence of suspected hepatic fibrosis among an obese adolescent population $^{(18)}$. For that reason, we then re-analyzed our data using $\geq 5.50 \mathrm{kPa}$ to indicate fibrosis, and found that the prevalence of suspected fibrosis remained similar to that found using a cutoff value of $\geq 5.10 \mathrm{kPa}$ $(53.7 \%, 36 / 67$ vs. $62.7 \%, 42 / 67$ patients, $p=0.29)$. All other data analyses using $5.50 \mathrm{kPa}$ remained similar when compared to our original analyses using $5.10 \mathrm{kPa}$, including mean APRI, which was significantly $(p=0.038)$ lower in the group without suspected fibrosis (Group A, $0.29 \pm 0.17$,

Tab. 3. Demographic and laboratory features of patients stratified by $A_{1 c}$

\begin{tabular}{|c|c|c|c|c|}
\hline & Entire cohort $N=67$ & $\begin{array}{c}\text { Group C } \\
\text { Normal } A_{1 \mathrm{c}} \\
(<5.7 \%) \\
N=35 \\
\text { Mean } \pm \text { SD (range) }\end{array}$ & $\begin{array}{c}\text { Group D } \\
\text { Abnormal } A_{1 c} \\
(\geq 5.7 \%) \\
N=32 \\
\text { Mean } \pm \text { SD (range) }\end{array}$ & $\begin{array}{c}P=\text { for Group } C \\
\text { vs. Group D }\end{array}$ \\
\hline Age (yr) & $\begin{array}{c}13.59 \\
(8-18) \\
\end{array}$ & $\begin{array}{c}12.97 \\
(8-18) \\
\end{array}$ & $\begin{array}{c}14.25 \\
(8-18) \\
\end{array}$ & 0.087 \\
\hline Gender & $\begin{array}{c}\text { Male }=35 \\
\text { Female }=32\end{array}$ & $\begin{array}{c}\text { Males }=18 \\
\text { Female }=14\end{array}$ & $\begin{array}{c}\text { Males }=17 \\
\text { Female }=18\end{array}$ & 0.529 \\
\hline BMI SDS & $\begin{array}{l}2.49 \pm 0.39 \\
(1.45-3.37)\end{array}$ & $\begin{array}{l}2.39 \pm 0.38 \\
(1.45-3.11)\end{array}$ & $\begin{array}{l}2.53 \pm 0.40 \\
(1.94-3.37)\end{array}$ & 0.169 \\
\hline Serum ALT (U/L) & $\begin{array}{c}78.48 \pm 44.75 \\
(19-189) \\
\end{array}$ & $\begin{array}{c}82.41 \pm 45.63 \\
(19-185) \\
\end{array}$ & $\begin{array}{c}74.16 \pm 44.10 \\
(23-189) \\
\end{array}$ & 0.462 \\
\hline Serum AST (U/L) & $\begin{array}{c}40.1 \pm 22.1 \\
(11-110) \\
\end{array}$ & $\begin{array}{c}40.09 \pm 18.54 \\
(19-110) \\
\end{array}$ & $\begin{array}{c}40.13 \pm 25.74 \\
(11-106) \\
\end{array}$ & 0.994 \\
\hline$A_{1 c}(\%)$ & $\begin{array}{c}5.77 \pm 0.64 \\
(4.5-7.9)\end{array}$ & $\begin{array}{c}5.36 \pm 0.23 \\
(4.5-5.6)\end{array}$ & $\begin{array}{c}6.19 \pm 0.66 \\
(5.7-7.9) \\
\end{array}$ & $<0.001$ \\
\hline SWE Score (kPa) & $\begin{array}{c}5.61 \pm 1.43 \\
(1.47-10.45)\end{array}$ & $\begin{array}{c}5.82 \pm 1.48 \\
(2.69-10.45) \\
\end{array}$ & $\begin{array}{l}5.39 \pm 1.36 \\
(1.47-9.85)\end{array}$ & 0.233 \\
\hline HOMA-IR & $\begin{array}{c}10.62 \pm 10.19 \\
(2.84-49.24)\end{array}$ & $\begin{array}{c}10.49 \pm 12.04 \\
(2.84-49.24) \\
\end{array}$ & $\begin{array}{l}10.84 \pm 6.47 \\
(4.61-25.25) \\
\end{array}$ & 0.940 \\
\hline Vitamin $\mathrm{D}, 25 \mathrm{OH}(\mathrm{ng} / \mathrm{mL})$ & $\begin{array}{c}14.23 \pm 5.44 \\
(6-28) \\
\end{array}$ & $\begin{array}{c}11.80 \pm 4.18 \\
(6-20)\end{array}$ & $\begin{array}{c}15.75 \pm 5.65 \\
(7-28) \\
\end{array}$ & 0.025 \\
\hline Platelets ( $\times 10^{9}$ per liter) & $\begin{array}{c}310 \pm 75.50 \\
(187-490)\end{array}$ & $\begin{array}{c}299.89 \pm 66.38 \\
(217-455) \\
\end{array}$ & $\begin{array}{c}326.46 \pm 76.01 \\
(187-490)\end{array}$ & 0.303 \\
\hline APRI & $\begin{array}{l}0.40 \pm 0.25 \\
(0.12-1.19)\end{array}$ & $\begin{array}{l}0.42 \pm 0.24 \\
(0.16-1.19) \\
\end{array}$ & $\begin{array}{c}0.36 \pm(0.28) \\
(0.12-1.01)\end{array}$ & 0.494 \\
\hline Metformin users & 8 & 1 patient & 7 patients & 0.016 \\
\hline
\end{tabular}




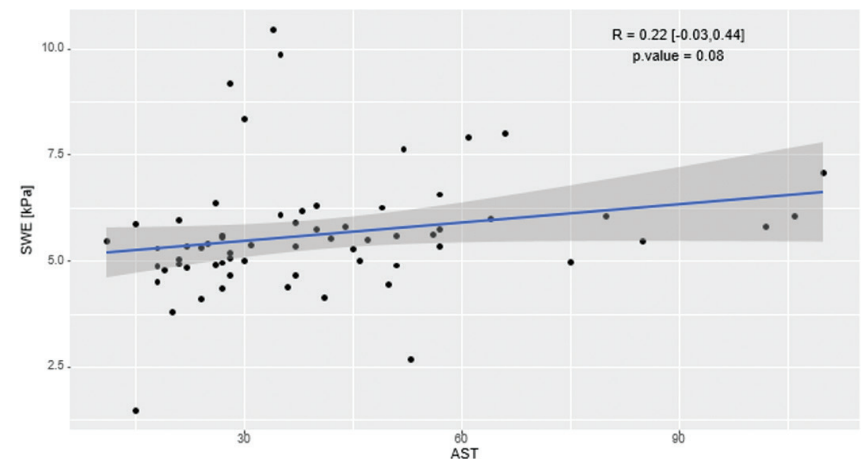

Fig. 1. The association between serum AST $(U / L)$ and SWE score as measured using SWE in $\mathrm{kPa}$. The overall association was not significant $(p=0.08)$ and there were several patients with elevation in SWE score, but normal AST, while others had marked elevations in AST, but no elevation in SWE score. AST - aspartate aminotransferase; SWE Score - liver stiffness measured in kilopascals $(\mathrm{kPa})$

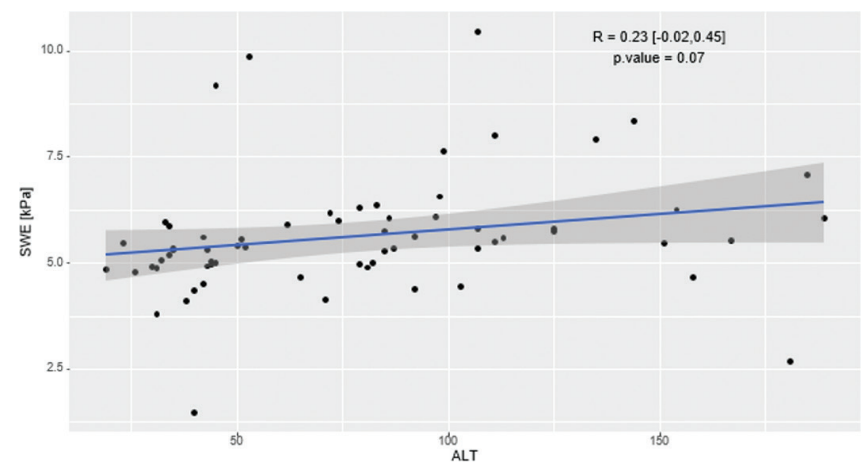

Fig. 2. The association between serum $A L T(U / L)$ and SWE score as measured using SWE in $\mathrm{kPa}$. The overall association was not significant $(\mathrm{p}=0.07)$ and there were several patients with elevation in SWE score, but normal ALT, while others had marked elevations in ALT, but no elevation in SWE score. ALT - alanine aminotransferase; SWE Score - liver stiffness measured in kilopascals ( $\mathrm{kPa}$ )

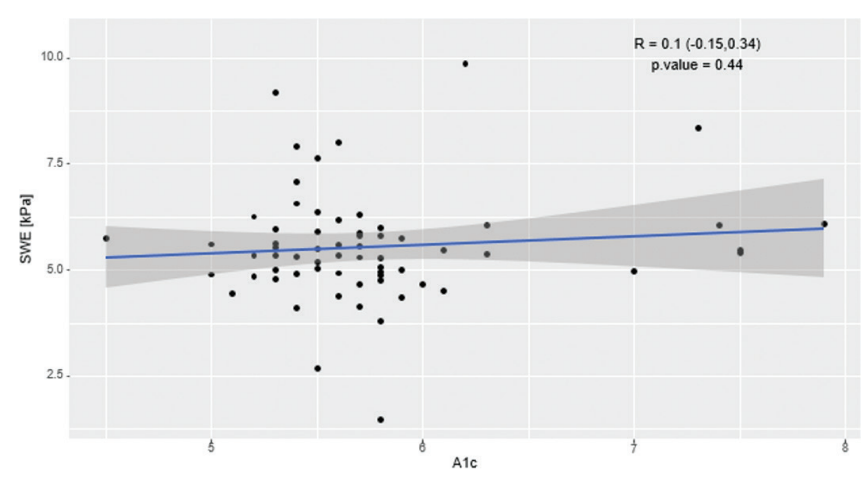

Fig. 3. The association between $A_{1 c}(\%)$ and SWE score as measured using SWE in $\mathrm{kPa}$. The overall association was not significant $(\mathrm{p}=0.44)$ and there were several patients with elevation in SWE score, but normal $A_{1 c}$, while others had marked elevations in $A_{1 c}$, but no elevation in SWE score. $A_{t c}-$ glycohemoglobin; SWE Score - liver stiffness measured in kilopascals $(\mathrm{kPa})$

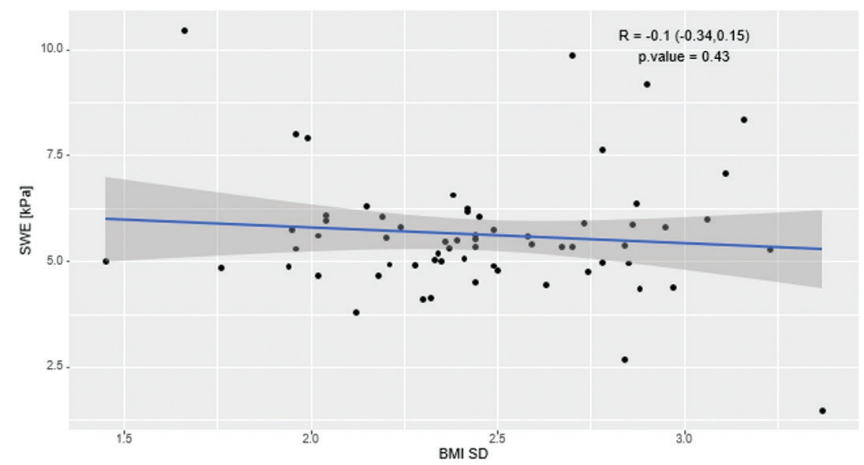

Fig. 4. The association between BMI SD and SWE score as measured using SWE in $\mathrm{kPa}$. The overall association was not significant $(\mathrm{p}=0.43)$ and there were several patients with elevation in SWE score, but only modest elevation in BMI $S D(1.5-2.0)$, while others had marked elevations in BMI-SD (2.0-3.5), but no elevation in SWE score. BMI SD - body mass index standard deviation score for age; SWE Scoreliver stiffness measured in kilopascals $(\mathrm{kPa})$

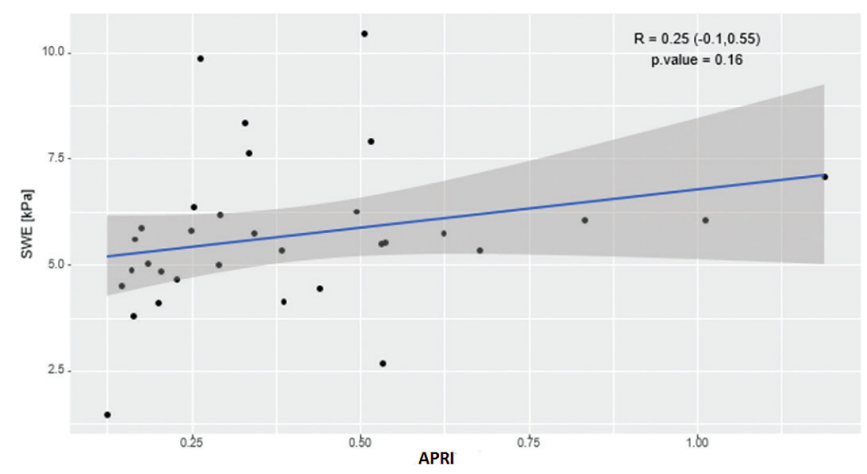

Fig. 5. The association between APRI and SWE score as measured using SWE in $\mathrm{kPa}$. The overall association was not significant $(\mathrm{p}=0.16)$ and there were several patients with elevation in SWE score, but normal APRI, while others had marked elevations in APRI, but no elevation in SWE score. SWE Score - liver stiffness measured in kilopascals (kPa); APRI aspartate aminotransferase (AST)/platelet ratio index

0.12-0.38) compared to the group with suspected fibrosis (Group B, $0.48 \pm 0.28,0.16-1.19$ ).

Next, we compared demographic and laboratory findings between patients with normal $A_{1 c}\left(A_{1 c}<5.7 \%\right.$, Group $\left.C\right)$ and those with elevated $A_{1 c}>5.7 \%$ (Group D). The latter included both those with pre-DM $\left(\mathrm{A}_{1 \mathrm{c}} 5.7-6.4 \%\right)$ and DM $\left(\mathrm{A}_{1 \mathrm{c}}>6.5 \%\right)$. As shown in Tab. 3, there were 35 patients with $A_{1 c}<5.7 \%$ (Group C). Group D included 32 patients with $A_{1 c} \geq 5.7 \%$. The mean SWE score for Group D $(5.39 \pm 1.36 \mathrm{kPa}$, range 1.47-9.85) was similar to that of Group C (5.82 $\pm 1.48 \mathrm{kPa}$, range 2.69-10.45, $p=0.233$ ). The mean serum ALT (Group $\mathrm{C}=82.41 \pm 45.63 \mathrm{U} / \mathrm{L}$, range $=19-185 ;$ Group $\mathrm{D}=74.16 \pm$ $44.10 \mathrm{U} / \mathrm{L}$, range $=23-189, p=0.462)$ and mean serum AST (Group $\mathrm{C}=40.09 \pm 18.54 \mathrm{U} / \mathrm{L}$, range $=19-110$; Group $\mathrm{D}=$ $40.13 \pm 25.74 \mathrm{U} / \mathrm{L}$, range $=11-106$ were also similar $(p=$ $0.994)$ between the two groups. There was also no difference between the two groups in the HOMA-IR or APRI. Serum 25-hydroxy vitamin D3 levels were significantly higher in Group D (15.75 $\pm 5.65 \mathrm{ng} / \mathrm{ml}$, range 7-28) when compared 
to Group C $(11.80 \pm 4.18 \mathrm{ng} / \mathrm{ml}$, range $6-20, p=0.025)$ but this was no longer significant when patients receiving vitamin D supplementation were excluded. As expected, a greater proportion of patients in Group D $(7 / 32,22 \%)$ were taking Metformin when compared to those in Group C (1/35, $2.9 \%, p=0.016)$.

We then analyzed the data for any significant associations between SWE score, $A_{1 c}$, BMI-SDS, serum AST or serum ALT across the entire cohort. As shown in Fig. 1 and Fig. 2, there were no significant associations between serum AST or ALT values and SWE score. As shown in Fig. 3 and Fig. 4, there were no significant associations between SWE score and $A_{1 c}$ or BMI-SDS. Finally, as shown in Fig. 5, there was no significant association between SWE score and APRI across the entire cohort.

\section{Discussion}

In this cohort of overweight or obese Hispanic youth, the prevalence of suspected hepatic fibrosis as determined by SWE was $62.7 \%$. Both TE and SWE are non-invasive measures of hepatic stiffness, a surrogate for liver fibrosis. However, we chose to use the technique of point SWE for several reasons. First, point SWE has a higher rate of reliable liver stiffness measurement than $\mathrm{TE}^{(23)}$. Second, SWE also has a lower failure rate than TE in obese patients. This has been attributed to the ability of SWE to use B-Mode (2-Dimensional) visualization, which allows the operator to select the proper site for reading, away from areas of obesity $^{(23)}$. In contrast, TE relies on the operator to blindly place the probe at the patient's mid-axillary plane.

Based on our current data, we believe the true prevalence of hepatic fibrosis in this high-risk population may be much greater than previously recognized. Several studies have documented that serum ALT and AST levels (markers for inflammation) fail to detect many patients with hepatic fibrosis or even cirrhosis and for that reason, serum ALT or AST levels are a specific but not sensitive assessment. We found a significant difference in serum AST and ALT when comparing patients with normal SWE score (Group A) and those with an elevated SWE score (Group B). The specificity of AST and ALT levels for identifying those at risk for hepatic fibrosis support our use of $\mathrm{SWE} \geq 5.10 \mathrm{kPa}$ as a cutoff to identify patients at high risk for hepatic fibrosis. However, we found no association between SWE and serum AST or ALT across the entire cohort, supporting previous observations that serum AST and ALT are not sensitive indicators of those who develop hepatic fibrosis and further suggest that SWE may be a more sensitive screening tool. We also found a significantly greater APRI in patients with suspected fibrosis (Group B), but failed to find a significant association between SWE score and APRI across the entire cohort. APRI has been used in adult patients to stage hepatic fibrosis in chronic liver conditions $^{(24)}$ and is believed by some to be a more sensitive test than serum AST alone. However, our data suggest that APRI might not be as sensitive an indicator in adolescents as it is for adults. Elevated APRI may also, however, be a specific indicator of hepatic fibrosis in this population.

Although obesity is a well-recognized adult risk factor for NAFLD, we did not find a significant association between BMI-SDS and suspected hepatic fibrosis detected by SWE. It remains highly likely that obesity is a major risk factor and that we failed to detect an association because all of our subjects were overweight or obese. Additional study of SWE in youth across a wider spectrum of BMI-SDS and abdominal circumference measurement will be required to address this question more directly.

T2DM is another known risk factor for hepatic fibrosis and some authors suggest that insulin resistance, a cardinal feature of T2DM, may be causative ${ }^{(11)}$. We found no significant association between SWE score and either $\mathrm{A}_{1 \mathrm{c}}$ as a measure of average glycemia or HOMA-IR as a measure of insulin resistance. It is important to note in this regard, that the number of subjects in our study is small and that HOMA-IR was not available for all subjects. Furthermore, given the degree of overweight and obesity, it is highly likely that all of our subjects may have had some degree of insulin resistance clouding this relationship.

Early identification of NAFLD is important in an attempt to identify youth at risk to develop NASH. Current adult guidelines recommend screening with an elastography method if NAFLD is suspected in order to stage the degree of suspected fibrosis and intervene appropriately. Adult scoring systems are unreliable for staging the degree of fibrosis in children with NAFLD and no staging guidelines currently exist for the pediatric population ${ }^{(17)}$. Given the high prevalence of NAFLD, improved methods for detection and staging are necessary. Despite the fact that serum AST, ALT, and APRI are not independent sensitive indicators of hepatic fibrosis, our data suggest that, when combined with SWE, they could be used to monitor youth at risk for NAFLD and NASH, allowing for early intervention.

Our study has several limitations. First and most importantly, we did not have histological confirmation of fibrosis in all of our patients. However, we did have liver biopsy in five patients from Group B, all of whom had fibrosis ranging from stage 1 to stage 2 . Second, there is a paucity of SWE data in youth. This limits the ability to select an SWE value $(\mathrm{kPa})$ which will optimize the positive and negative predictive value of this procedure. We chose $<5.10 \mathrm{kPa}$ to distinguish lack of fibrosis from suspected fibrosis $(\geq 5.10 \mathrm{kPa})$ based on a previous study ${ }^{(7)}$. This study was limited to an investigation of Italian youth and may or may not be applicable to a more diverse racial and ethnic population. However, we had similar findings when we reanalyzed our data using $5.50 \mathrm{kPa}$, which was suggested in a Korean study ${ }^{(18)}$. Third, ours is a pilot cohort limited to 67 Hispanic youth referred to our obesity clinic and HOMA-IR was not available for all subjects. Finally, this study is limited to youth who underwent SWE at our academic institution, which might have introduced selection bias into our population. 


\section{Conclusion}

In conclusion, using SWE, we found a high prevalence $(62.7 \%)$ of suspected hepatic fibrosis in a group of highrisk, overweight or obese Hispanic youth. We also found significant elevations in serum AST, ALT, and APRI in patients with suspected fibrosis $(\geq 5.10 \mathrm{kPa})$. However, we found no significant association between SWE score and serum AST, ALT or APRI across the entire cohort. Our study confirms the feasibility of SWE as a non-invasive

\section{References}

1. Skinner AC, Ravanbakht SN, Skelton JA, Perrin EM, Armstrong SC: Prevalence of obesity and severe obesity in US children, 1999-2016. Pediatrics 2018; 141: e20173459.

2. Dyson JK, McPherson S, Anstee QM: Non-alcoholic fatty liver disease: non-invasive investigation and risk stratification. J Clin Pathol 2013; 66: 1033-1045.

3. Goyal NP, Schwimmer JB: The genetics of pediatric nonalcoholic fatty liver disease. Clin Liver Dis 2018; 22: 59-71.

4. Alisi A, Feldstein AE, Villani A, Raponi M, Nobili V: Pediatric nonalcoholic fatty liver disease: A multidisciplinary approach. Nat Rev Gastroenterol Hepatol 2012; 9: 152-161.

5. Pan JJ, Fallon MB: Gender and racial differences in nonalcoholic fatty liver disease. World J Hepatol 2014; 6: 274-283.

6. Anderson EL, Howe LD, Jones HE, Higgins JPT, Lawlor DA, Fraser A: The prevalence of non-alcoholic fatty liver disease in children and adolescents: A systematic review and meta-analysis. PloS One 2015; 10: e0140908.

7. Nobili V, Vizzutti F, Arena U, Abraldes JG, Marra F, Pietrobattista A et al.: Accuracy and reproducibility of transient elastography for the diagnosis of fibrosis in pediatric nonalcoholic steatohepatitis. Hepatology 2008; 48: 442-448.

8. Armstrong T, Ly KV, Murthy S, Ghahremani S, Kim GH, Calkins KL et al.: Free-breathing quantification of hepatic fat in healthy children and children with nonalcoholic fatty liver disease using a multi-echo 3-D stack-of-radial MRI technique. Pediatr Radiol 2018; 48: 941-953.

9. Shimizu M, Suzuki K, Kato K, Jojima T, Iijima T, Murohisa T et al.: Evaluation of the effects of dapagliflozin, a sodium-glucose co-transporter-2 inhibitor, on hepatic steatosis and fibrosis using transient elastography in patients with type 2 diabetes and non-alcoholic fatty liver disease. Diabetes Obes Metab 2019; 21: 285-292.

10. Tolman KG, Fonseca V, Dalpiaz A, Tan MH: Spectrum of liver disease in type 2 diabetes and management of patients with diabetes and liver disease. Diabetes Care 2007; 30: 734-743.

11. Byrne CD, Targher G: NAFLD: a multisystem disease. J Hepatol 2015; 62 Suppl: S47-S64.

12. Trombetta M, Spiazzi G, Zoppini G, Muggeo M: Review article: type 2 diabetes and chronic liver disease in the verona diabetes study. Aliment Pharmacol Ther 2005; 22 Suppl: 24-27.

13. de Lédinghen V, Le Bail B, Rebouissoux L, Fournier C, Foucher J, Miette $\mathrm{V}$ et al.: Liver stiffness measurement in children using FibroScan: Feasibility study and comparison with fibrotest, aspartate transaminase method to identify youth with suspected hepatic fibrosis and broadens the ethnic groups that have been studied.

\section{Conflict of interest}

The authors involved in the submitted work declare that the study was completed without any commercial or financial relationships that could be construed as a potential conflict of interest.

to platelets ratio index, and liver biopsy. J Pediatr Gastroenterol Nutr 2007; 45: 443-450.

14. Kim E, Kang Y, Hahn S, Lee MJ, Park YN, Koh H: The efficacy of aspartate aminotransferase-to-platelet ratio index for assessing hepatic fibrosis in childhood nonalcoholic steatohepatitis for medical practice. Korean J Pediatr 2013; 56: 19-25.

15. Shah J, Okubote T, Alkhouri N: Overview of updated practice guidelines for pediatric nonalcoholic fatty liver disease. Gastroenterol Hepatol (N Y) 2018; 14: 407-414.

16. Fallatah HI, Akbar HO, Fallatah AM: Fibroscan compared to FIB4, APRI, and AST/ALT ratio for assessment of liver fibrosis in Saudi patients with nonalcoholic fatty liver disease. Hepat Mon 2016; 16: e38346.

17. Mansoor S, Yerian L, Kohli R, Xanthakos S, Angulo P, Ling S et al.: The evaluation of hepatic fibrosis scores in children with nonalcoholic fatty liver disease. Dig Dis Sci 2015; 60: 1440-1447.

18. Joo I, Kim SY, Park HS, Lee ES, Kang HJ, Lee JM: Validation of a new point shear-wave elastography method for noninvasive assessment of liver fibrosis: a prospective multicenter study. Korean J Radiol 2019; 20: 1527-1535.

19. Lee CK, Perez-Atayde AR, Mitchell PD, Raza R, Afdhal NH, Jonas MM: Serum biomarkers and transient elastography as predictors of advanced liver fibrosis in a united states cohort: the boston children's hospital experience. J Pediatr 2013; 163: 1058-1064.e2.

20. Dhyani M, Gee MS, Misdraji J, Israel EJ, Shah U, Samir AE: Feasibility study for assessing liver fibrosis in paediatric and adolescent patients using real-time shear wave elastography. J Med Imaging Radiat Oncol 2015; 59: 687-694.

21. Ferraioli G, Filice C, Castera L, Choi BI, Sporea I, Wilson SR et al.: WFUMB guidelines and recommendations for clinical use of ultrasound elastography: part 3: liver. Ultrasound Med Biol 2015; 41: 1161-1179.

22. ADA. American Diabetes Association Website. https://www.diabetes.org/ a1c/diagnosis. Accessed: 6.01.2020.

23. Osman AM, El Shimy A, Abd El Aziz MM: 2D shear wave elastography (SWE) performance versus vibration-controlled transient elastography (VCTE/fibroscan) in the assessment of liver stiffness in chronic hepatitis. Insights Imaging 2020; 11: 38.

24. Lin ZH, Xin YN, Dong QJ, Wang Q, Jiang XJ, Zhan SH et al.: Performance of the aspartate aminotransferase-to-platelet ratio index for the staging of hepatitis C-related fibrosis: an updated meta-analysis. Hepatology 2011; 53: 726-736. 